\title{
Parallax programs at the Bordeaux Observatory at sub-mas level accuracy
}

\section{Ducourant ${ }^{1}$, R. Teixeira ${ }^{2}$, J.-F. le Campion ${ }^{1}$ and G. Chauvin ${ }^{3}$}

${ }^{1}$ Observatoire Aquitain des Sciences de l'Univers, CNRS-UMR 5804, BP 89, 33270 Floirac,

France. email: ducourant@obs.u-bordeaux1.fr, email: lecampion@obs.u-bordeaux1.fr

${ }^{2}$ Instituto de Astronomia, Geofísica e Ciências Atmosféricas, Universidade de São Paulo, Rua do Matão, 1226 - Cidade Universitária, 05508-900 São Paulo - SP, Brasil. email:

teixeira@astro.iag.usp.br

${ }^{3}$ Laboratoire d'Astrophysique Observatoire de Grenoble BP 53 F-38041 GRENOBLE Cdex 9, France. email: Gael.Chauvin@obs.ujf-grenoble.fr

\begin{abstract}
We present ongoing parallax programs developed at the Bordeaux Observatory several years ago. We describe the necessary steps leading to sub-milliarcsecond accuracy of calculated parallax. We show the importance of global methods for accurate parallax determination.
\end{abstract}

Keywords. astrometry, stars: distances, solar neighborhood, methods: data analysis

\section{Introduction}

With the increasing interest in extra-solar planets, parallax measurements appear to be crucial since most of known stars hosting a planet are situated close to the Sun $(d \leqslant 100 p c)$ and therefore accessible to ground-based astrometry.

The use of $4 \mathrm{~m}$-class telescopes allows to derive parallaxes with sub-milli-arcsecond accuracy after 2 years of regular observations. In such a small time-base programs with so high accuracy, it is important to minimize all potential sources of errors and biases and to realistically evaluate the errors, and their origins.

We present here the Bordeaux parallax programs, describing several steps leading to high accuracy parallax determination. We investigate through Monte-Carlo simulations the achievable precision on parallaxes for ground-based observations using global iterative methods (central overlap methods Eichhorn (1997)). We present the dependency of errors on the reference catalogue used to calibrate the CCD and on the star density of the field (number of objects participating in the global fit).

\section{The Bordeaux Parallax Programs}

In the last years, we developed in Bordeaux several programs of parallax measurements aiming at the characterisation of the target through distance determination :

- Systematic search of nearby stars in wide field survey : Ducourant et al. (1998).

- Free Floating Brown dwarfs characterisation : Hawkins et al. (1998).

- Halo High velocity white dwarf candidates segregation : Ducourant et al. (2007a).

- An accurate distance and mass determination for the first imaged planetary system 2M1207Ab : Ducourant et al. (2007b).

- Distance determination to the TW Hydrae association, under realisation (see Teixeira et al. in this volume for details).

For these programs we developed a code of global iterative treatment (Eichhorn 1997) of the observations which take advantage of any well-measured star in each image to 
participate in the global fit, allowing to define a re-inforced reference system with respect to which the target parallax can be defined.

\section{Methodology}

To reach sub-milliarcsecond accuracies, it is important to minimize each source of bias and error. The major steps are :

- The observation strategy ( $\mathrm{S} / \mathrm{N}$ of objects, frequency, number of repeated frames, distance to zenith, filter, length of program). It appears that a crucial point concerns the zenith distance which has to be minimized in order to minimize differential colour refraction effects between target and field stars. A large number of frames taken at each epoch appears important for a correct evaluation of the errors.

- The selection of reference stars used to calibrate pixel scale and orientation of CCD (number, repartition in the field, catalogue astrometry) is an important step. It appears that a catalogue as $2 \mathrm{MASS}$ is suited for that purpose although it is not a proper motion catalogue and that its central epoch is 2000.0. We observe that it introduces less bias in the astrometry than the use of UCAC2, more accurate and with proper motion but so poorly dense (See also I. Platais, same volume, for needs of Deep Astrometric Standards).

- The global iterative resolution with a large number of objects participating to the global solution (fit stars) (also important to average differential colour refraction effects) to correctly evaluate the correction from relative to absolute parallax.

\section{Results}

We show through numerous Monte-Carlo simulations that at the sub-mas level the target's measured parallax is strongly dependent on the number of reference stars and that methods (conventional or global) that rely on low number of fit stars may result in a biased solution.

\section{References}

Ducourant, C., Teixeira R., Hambly N. et al., 2007a, A\&GA, in press.

Ducourant, C., Teixeira R., Chauvin, G., Daigne, G., Le Campion, J. F., Song, I., \& Zuckerman, B., 2007b, $A \mathscr{E} A$, submitted.

Ducourant, C., Dauphole, B., Rapaport, M., Colin, J., \& Geffert, M., 1998, A\&\&A, 333, 882.

Eichhorn, H., 1997, Astron. Astrophys., 327, 404.

Hawkins, M. R. S., Ducourant, C., Rapaport, M., \& Jones, H. R. A., 1998, MNRAS,294, 505. 